OPEN ACCESS

Edited by:

Qiongjie Zhou,

Fudan University, China

Reviewed by:

Rajakumar Anbazhagan,

Eunice Kennedy Shriver National Institute of Child Health and Human Development (NICHD), United States Irene Scavello,

Careggi University Hospital, Italy

*Correspondence: John D. Meeker meekerj@umich.edu

Specialty section: This article was submitted to

Reproduction, a section of the journa

Frontiers in Endocrinology

Received: 15 July 2021 Accepted: 25 August 2021 Published: 15 September 2021

Citation:

Cathey AL, Watkins DJ, Rosario ZY, Vega CMV, Mukherjee B, O'Neill MS, Loch-Caruso R, Alshawabkeh AN, Cordero JF and Meeker JD (2021) Gestational Hormone Concentrations Are Associated With Timing of Delivery in a Fetal Sex-Dependent Manner.

Front. Endocrinol. 12:742145. doi: 10.3389/fendo.2021.742145

\section{Gestational Hormone Concentrations Are Associated With Timing of Delivery in a Fetal Sex-Dependent Manner}

\author{
Amber L. Cathey ${ }^{1}$, Deborah J. Watkins ${ }^{1}$, Zaira Y. Rosario ${ }^{2}$, Carmen M. Vélez Vega ${ }^{2}$, \\ Bhramar Mukherjee ${ }^{3}$, Marie S. O'Neill ${ }^{1,4}$, Rita Loch-Caruso ${ }^{1}$, Akram N. Alshawabkeh $^{5}$, \\ José F. Cordero ${ }^{6}$ and John D. Meeker ${ }^{1 *}$ \\ ${ }_{1}^{1}$ Department of Environmental Health Sciences, University of Michigan School of Public Health, Ann Arbor, MI, United \\ States, ${ }^{2}$ Graduate School of Public Health, University of Puerto Rico, San Juan, PR, United States, ${ }^{3}$ Department of \\ Biostatistics, University of Michigan School of Public Health, Ann Arbor, MI, United States, ${ }^{4}$ Department of Epidemiology, \\ University of Michigan School of Public Health, Ann Arbor, MI, United States, ${ }^{5}$ College of Engineering, Northeastern \\ University, Boston, MA, United States, ${ }^{6}$ College of Public Health, University of Georgia, Athens, GA, United States
}

Background: Early delivery remains a significant public health problem that has longlasting impacts on mother and child. Understanding biological mechanisms underlying timing of labor, including endocrine disruption, can inform prevention efforts.

Methods: Gestational hormones were measured among 976 women in PROTECT, a longitudinal birth cohort in Puerto Rico. We evaluated associations between hormone concentrations at 18 and 26 weeks gestation and gestational age at birth, while assessing effect modification by fetal sex. Exploratory analyses assessed binary outcomes of overall preterm birth (PTB, $<37$ weeks gestation) and the spontaneous PTB subtype, defined as preterm premature rupture of membranes, spontaneous preterm labor, or both. Multivariable logistic and linear regressions were fit using visit-specific hormone concentrations, and fetal sex-specific effects were estimated using interaction terms. Main outcome models were adjusted for maternal age, education, marital status, alcohol consumption, environmental tobacco smoke exposure, and pre-pregnancy body mass index (BMI). Exploratory models adjusted for maternal age and education.

Results: We observed reduced gestational age at birth with higher circulating $\mathrm{CRH}$ ( $\beta$ : -2.73 days, $95 \% \mathrm{Cl}:-4.97,-0.42)$, progesterone ( $\beta$ : -4.90 days, $95 \% \mathrm{Cl}:-7.07,-2.73)$, and fT4 concentrations ( $\beta$ : -2.73 days, $95 \% \mathrm{Cl}:-4.76,-0.70)$ at 18 weeks specifically among male fetuses. Greater odds of overall and spontaneous PTB were observed among males with higher $\mathrm{CRH}$, estriol, progesterone, total triiodothyronine (T3), and free thyroxine ( $\mathrm{fT} 4$ ) concentrations. Greater odds of PTB among females was observed with higher testosterone concentrations. 
Conclusions: Various associations between hormones and timing of delivery were modified by fetal sex and timing of hormone measurement. Future studies are needed to understand differential mechanisms involved with timing of labor between fetal sexes.

Keywords: birth cohort, corticotropin releasing hormone, gestational age, pregnancy, preterm birth, progesterone

\section{INTRODUCTION}

Delivery before 37 weeks of gestation, or preterm birth (PTB), is the leading cause of neonatal mortality worldwide (1). The preterm birth rate in the United States is higher than that of most other high-income nations and increased to $9.85 \%$ from 2014 to 2016 (2). This increase was primarily driven by late PTBs, which occur between 34 and 36 weeks gestation, suggesting that development of targeted prediction strategies for detecting pregnancies destined for late preterm delivery could have marked impacts on the public health burden of early delivery. Infants born preterm are at increased risk for adverse health outcomes later in life including reduced renal function (3), neurodevelopmental impairments (4), cerebral palsy (5), and reduced myocardial function (6). Despite being a common public health problem, the causes of early delivery are largely unknown.

Preterm birth can be subcategorized into spontaneous and indicated groups based on obstetric presentation, and some have suggested that assessing these subtypes separately may better inform their etiologies. The spontaneous subtype occurs from spontaneous premature initiation of labor or rupture of membranes, and is characterized by an inflammatory uterine environment which is not present in medically indicated PTBs (7). The physiological changes that occur upstream of clinical detection of PTB may be distinct between subgroups and could help clinicians determine pregnancies that are at high risk for early delivery.

The maternal and fetal endocrine milieus change and interact in unique ways at different points throughout gestation. Numerous hormones play critical roles during pregnancy and so understanding their effects on the uterine environment and fetal growth could shed light on mechanisms involved with early delivery. Hormones of special interest during pregnancy can be broken into two categories: reproductive hormones and thyroid hormones. Reproductive hormones include testosterone, sex hormone binding globulin (SHBG), estriol, and progesterone. Elevated concentrations of testosterone are observed in women with polycystic ovarian syndrome (PCOS), as well as women with recurring miscarriages. Testosterone may reduce levels of endometrial secretory proteins which are positively associated with length of gestation (8), or it may act antagonistically with estrogens (9). Testosterone circulates through the body bound to SHBG, so changing SHBG concentrations could affect the concentration of bioavailable testosterone. The coordination of concentrations of estriol and progesterone are critically important for the timing of labor, as estriol primes the uterus for contractions (10) and progesterone promotes quiescence of the uterus until the time of labor $(11,12)$. Progesterone keeps the actions of estriol in check through pregnancy, but an untimely shift in dominance from progesterone to estriol could result in early delivery.

Thyroid hormones are critical early in pregnancy for proper brain and skeletal development of the fetus (13). The maternal supply of thyroxine (T4) is particularly important in the first half of pregnancy, before the fetal thyroid gland has matured enough to produce adequate hormones (14). Through gestation, thyroid hormones are important for fetal growth and are correlated with infant weight and length at birth (15). Previous studies have demonstrated associations between clinical hyper- and hypothyroidism and adverse birth outcomes (16-19), but much less is known about subclinical thyroid disruption and gestational length.

Finally, corticotropin releasing hormone (CRH), which is secreted from the hypothalamus and normally involved in stress response, may also be key in understanding the endocrine role in early delivery. CRH concentrations are low in the first half of pregnancy and then begin to exponentially increase around the $20^{\text {th }}$ week of gestation to peak at birth (20). An earlier and more rapid increase of CRH concentrations has been observed in women who experience early delivery (21), suggesting that $\mathrm{CRH}$ may be involved in a placental clock and that monitoring $\mathrm{CRH}$ concentrations early in pregnancy could provide clues about pregnancies at risk for early delivery.

Few large epidemiological studies exist that assess a wide array of hormone concentrations and timing of delivery. The majority of existing research focuses on one hormone/class of hormones, which makes it challenging to gain a broad understanding of the endocrine pathways implicated in the etiology of early delivery (22-24). Importantly, the spontaneous subtype of PTB has not been well studied. Few previous studies have investigated hormone concentrations at more than one time point during gestation, which is critical because some hormone concentrations change dramatically throughout gestation. Previous work also has not widely assessed the impact of fetal sex on these associations, despite existing literature showing male fetuses conferring greater risk of early delivery, perhaps via a greater pro-inflammatory environment (25) or relatively greater weight for gestational age at birth (26). Further, concentrations of hormones such as testosterone, estriol, and progesterone have been shown to be influenced by the sex of the fetus (27-29). Because of these gaps in the literature, the aim of this study was to investigate associations between various hormone concentrations and gestational age at birth, specific to timing of hormone assessment and fetal sex. Additionally, we conducted an exploratory analysis in which binary outcomes of overall PTB and spontaneous PTB were assessed. 


\section{MATERIALS AND METHODS}

\section{Study Population}

Pregnant women were recruited into the PROTECT birth cohort between 2011 and 2018 at $14 \pm 2$ weeks' gestation from seven hospitals and prenatal clinics in northern Puerto Rico. Study design and recruitment protocols have been described elsewhere (30). Briefly, women were recruited at $14 \pm 2$ weeks gestation and were eligible to participate if they were between the ages of 18 and 40 years, participated in their first clinic visit before their $20^{\text {th }}$ week of pregnancy, had not taken oral contraceptives within 3 months of getting pregnant, had not used in vitro fertilization to get pregnant, and had no known preexisting medical or obstetric conditions. Demographic and self-reported health information was provided at the first clinic visit. This study was approved by the research and ethics committees of the University of Michigan School of Public Health, University of Puerto Rico, Northeastern University, and participating hospitals and clinics. All methods reported in this study were performed in accordance with relevant guidelines and regulations imposed by those institutions. All study participants provided full informed consent prior to participation.

\section{Hormone Measurements}

All women provided serum samples at their first and third clinic visits, aligning with median 18 (range 16-20) and 26 (range 2428) weeks gestation which are periods of rapid fetal growth and development. Serum samples were analyzed at the Central Ligand Assay Satellite Services (CLASS) laboratory in the Department of Epidemiology at the University of Michigan School of Public Health. Progesterone, sex hormone-binding globulin (SHBG), testosterone, total triiodothyronine (T3), total thyroxine (T4), free thyroxine (fT4), and thyroid-stimulating hormone (TSH) were measured using a chemiluminescence immunoassay. Estriol and corticotropin releasing hormone $(\mathrm{CRH})$ were measured using an enzyme immunoassay. Some hormone concentrations were not available for all participants due to sample volume limitations. The ratios of progesterone to estriol (Prog/E3) and T3 to T4 (T3/T4) were assessed in addition to measured hormones because of previous research indicating that the ratios may be better indices of gestational age at birth than single hormone measurements $(23,31,32)$. All hormone concentrations below the limit of detection (LOD) were replaced by the LOD divided by the square root of two.

\section{Birth Outcome Assessment}

Based on recommendations from the American College of Obstetricians and Gynecologists, self-reported date of the last menstrual period was collected at the first study visit and used in combination with early ultrasound measurements to determine gestational age at birth (33). Briefly, the LMP was used as the gold standard and was compared to ultrasound measurements taken primarily before 14 weeks gestation. Gestational age was changed from the LMP estimate to the ultrasound estimate if the difference between the two methods was greater than a certain number of days, depending on which week the ultrasound was performed. PTB was defined as delivery before 37 weeks gestation. We also assessed spontaneous PTB, defined as PTB presenting with premature rupture of membranes, spontaneous preterm labor, or both (7).

\section{Statistical Methods}

Distributions of demographic, health, and pregnancy characteristics were calculated. Distributions of hormone concentrations were assessed individually at each study visit. Summary measures of gestational hormone concentrations were also assessed for descriptive purposes using arithmetic means of all available concentrations for each study participant, or geometric means for log-normally distributed hormones. Intraclass correlation coefficients (ICCs) were used to assess between- and within-individual variability of hormone concentrations across study visits.

Linear regression was used to model gestational age at birth, and logistic regression was used to model overall and spontaneous PTB. All statistical models utilized visit-specific hormone concentrations as opposed to summary measures. Effect estimates specific to fetal sex and study visit were derived using two interaction terms: one between the exposure variable and an indicator for fetal sex, and one between the exposure variable and an indicator for study visit. Interaction term p-values less than 0.05 were deemed significant. Sandwich estimators were used in these models to correct for biased standard errors due to the non-repeating nature of outcome variables. Possible confounders were first explored by evaluating their associations with exposure and outcome variables. Then, possible confounders were added into statistical models in a forward stepwise manner and retained in the model if the resultant change in the main effect estimate was greater than $10 \%$. Main gestational age at birth models adjusted for categorical forms of maternal age, maternal education, marital status, exposure to environmental tobacco smoke, alcohol consumption, and pre-pregnancy BMI. All models assessing testosterone also included SHBG to adjust for bound testosterone. In exploratory analyses assessing binary outcomes of overall and spontaneous PTB, statistical models were adjusted only for maternal age and maternal education to maximize sample size.

\section{RESULTS}

Demographics of the study population are shown in Table $\mathbf{1}$. The majority of mothers were under the age of 30 (67.1\%), had at least some college education (79\%), were employed (63\%), had an annual household income under $\$ 30,000$ (63.1\%), were married $(53.1 \%)$, reported never smoking $(86 \%)$ or being exposed to environmental tobacco smoke (88.7\%), did not drink alcohol during pregnancy (93.6\%), had given birth to less than 2 previous children (86.9\%), and had a pre-pregnancy BMI of less than 25 (56.1\%).

Distributions of hormone concentrations are shown in Supplementary Table S1. ICCs for most hormones were high (0.65-0.86), indicating only modest variability in hormone measurements within individuals across study visits. 
TABLE 1 | Maternal demographic characteristics of the study population $(\mathrm{N}=976)$.

\begin{tabular}{|c|c|}
\hline & $\mathbf{N}(\%)$ \\
\hline \multicolumn{2}{|l|}{ Maternal Age (years) } \\
\hline $18-24$ & $354(36.3 \%)$ \\
\hline $25-29$ & 301 (30.8\%) \\
\hline $30-34$ & $206(21.1 \%)$ \\
\hline $35-41$ & $115(11.8 \%)$ \\
\hline \multicolumn{2}{|l|}{ Maternal Education } \\
\hline GED or less & 203 (21\%) \\
\hline Some College & $331(34.2 \%)$ \\
\hline Bachelors or Higher & $433(44.8 \%)$ \\
\hline \multicolumn{2}{|l|}{ Employment Status } \\
\hline No & 357 (37\%) \\
\hline Yes & 608 (63\%) \\
\hline \multicolumn{2}{|c|}{ Annual Household Income } \\
\hline$<10 \mathrm{k}$ & 269 (31.6\%) \\
\hline $10 \mathrm{k}-<30 \mathrm{k}$ & 268 (31.5\%) \\
\hline $30 \mathrm{k}-<50 \mathrm{k}$ & $203(23.8 \%)$ \\
\hline$>=50 \mathrm{k}$ & $112(13.1 \%)$ \\
\hline \multicolumn{2}{|l|}{ Marital Status } \\
\hline Single & 197 (20.4\%) \\
\hline Married & $521(53.9 \%)$ \\
\hline Cohabitating & $249(25.7 \%)$ \\
\hline \multicolumn{2}{|l|}{ Smoking Status } \\
\hline Never & $833(86 \%)$ \\
\hline Ever & $121(12.5 \%)$ \\
\hline Current & $15(1.55 \%)$ \\
\hline \multicolumn{2}{|c|}{ Daily Environmental Tobacco Smoke Exposure } \\
\hline Never & $808(88.7 \%)$ \\
\hline 1 Hour or less & $40(4.39 \%)$ \\
\hline$>1$ Hour & $63(6.92 \%)$ \\
\hline \multicolumn{2}{|l|}{ Alcohol Use } \\
\hline Never & $504(52.2 \%)$ \\
\hline Yes, before Pregnancy & $400(41.4 \%)$ \\
\hline Yes, currently & $62(6.42 \%)$ \\
\hline \multicolumn{2}{|c|}{ Number of Previous Children } \\
\hline 0 & $355(42.7 \%)$ \\
\hline 1 & $367(44.2 \%)$ \\
\hline 2 to 5 & $109(13.1 \%)$ \\
\hline \multicolumn{2}{|l|}{ Pre-Pregnancy BMI } \\
\hline$[0,25]$ & $520(56.1 \%)$ \\
\hline$(25,30]$ & $240(25.9 \%)$ \\
\hline Above 30 & 167 (18\%) \\
\hline \multicolumn{2}{|l|}{ Fetal Sex } \\
\hline Female & $464(48 \%)$ \\
\hline Male & $502(52 \%)$ \\
\hline
\end{tabular}

Exceptions were estriol (ICC: $-0.22,95 \%$ CI: $-0.35,-0.11$ ) and progesterone (ICC: $0.07,95 \%$ CI: $-0.04,0.17$ ), both of which displayed significantly higher concentrations at 26 weeks compared to 18 weeks.

Distributions of birth outcomes, stratified by fetal sex, are shown in Table 2. The median gestational age at birth was 39.1 weeks for pregnancies carrying either a male or a female fetus. Both overall and spontaneous PTB occurred more frequently among pregnancies with a male fetus $(10.7 \%$ and $6.6 \%$, respectively) than those with a female fetus ( $8.9 \%$ and $4.9 \%$, respectively).

\section{CRH and Reproductive Hormones}

Figure $\mathbf{1 A}$ shows the associations between $\mathrm{CRH}$ and reproductive hormone concentrations and gestational age at birth, specific to fetal sex and study visit (effect estimates,
TABLE 2 | Distributions of gestational age at birth, overall preterm birth, and spontaneous preterm birth.

\begin{tabular}{lccccccc}
\hline $\begin{array}{l}\text { Gestational Age } \\
\text { (wks) }\end{array}$ & Min & 10th & 25th & 50th & 75th & 90th & Max \\
\hline Male Fetuses & 23.3 & 36.6 & 38.3 & 39.1 & 40.0 & 40.7 & 42.7 \\
Female Fetuses & 21.1 & 37.0 & 38.1 & 39.1 & 40.1 & 40.7 & 42.7 \\
& Male & Female & & & & \\
& Fetuses & Fetuses & & & & \\
Preterm Birth & $\mathbf{N}(\%)$ & $\mathbf{N}(\%)$ & & & & \\
Yes & $53(10.7 \%)$ & $41(8.9 \%)$ \\
$\quad$ No & $444(89.3 \%)$ & $419(91.1 \%)$ \\
Spontaneous & $\mathbf{N}(\%)$ & $\mathbf{N}(\%)$ & & & & \\
Preterm Birth & $32(6.6 \%)$ & $22(4.9 \%)$ \\
Yes & $451(93.4 \%)$ & $428(95.1 \%)$ \\
No & & & & & & \\
\end{tabular}

confidence intervals, and p-values for fetal sex interaction terms can be found in Supplementary Table S2). Fetal sex significantly modified the association between progesterone and gestational age at birth (interaction $\mathrm{p}=0.015$ ); among male fetuses only, an IQR increase in progesterone at 18 weeks was associated with a decrease in gestational age at delivery of 4.9 days (95\% CI: 2.73, 7.07). Other significant findings were observed without significant effect modification by fetal sex. An IQR increase in CRH among male fetuses at 18 weeks was associated with a reduction in gestational age at delivery of 2.73 days (95\% CI: 0.42, 4.97). Male fetuses also had a marginal reduction in gestational age at birth with an IQR increase in the ratio of progesterone to estriol at both visits (visit $1 \beta:-1.75$ days, 95\% CI: -3.5, -0.07; visit $3 \beta:-2.24$ days, $95 \%$ CI: $-4.27,-0.28)$. Finally, female fetuses experienced an increase in gestational age at birth of 3.92 days (95\% CI: 0.77, 7.07) with an IQR increase in estriol at 26 weeks.

\section{Thyroid Hormones}

Figure 1B shows the associations between thyroid hormone concentrations and gestational age at birth, specific to fetal sexes and study visits (effect estimates, confidence intervals, and p-values for fetal sex interaction terms can be found in Supplementary Table S2). We did observe differences in these associations between fetal sexes, though none of them reached statistical significance. Generally, greater reductions in gestational age at birth were observed among male fetuses, but the only finding to reach statistical significance was for fT4 at 18 weeks ( $\beta$ : -2.73 days, 95\% CI: $-4.76,-0.70)$.

\section{Exploratory Analyses of Binary Outcomes}

Results for exploratory analyses assessing the binary outcomes of overall and spontaneous PTB and their associations with $\mathrm{CRH}$ and reproductive hormones are shown in Figure 2 (effect estimates, confidence intervals, and p-values for fetal sex interaction terms can be found in Supplementary Table S2). Fetal sex effect modification was much more apparent for these outcomes than for continuous gestational age at delivery. The associations between $\mathrm{CRH}$ and both overall and spontaneous PTB were significantly modified by fetal sex (interaction $\mathrm{p}=0.002$ and 0.003 , respectively); effect estimates were inverse among female fetuses and positive among male fetuses for both 
A

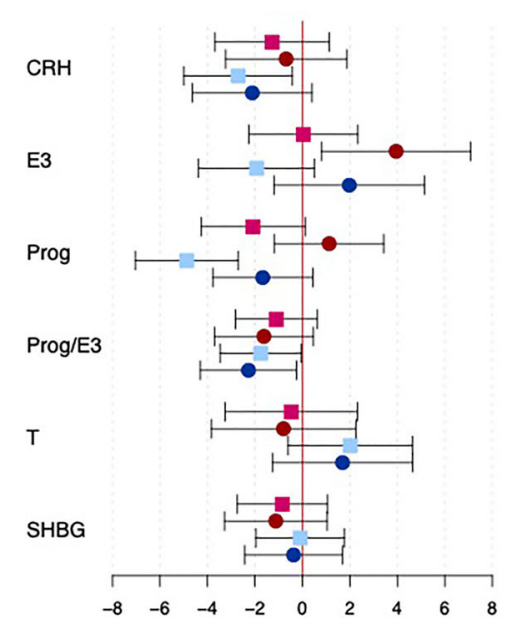

B

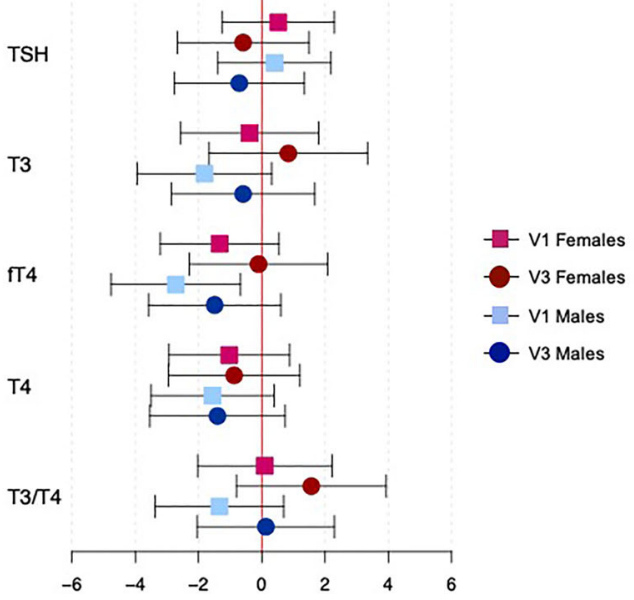

FIGURE 1 | Associations between hormone concentrations and gestational age at delivery, specific to fetal sexes and study visit. Panel (A) shows CRH and reproductive hormones, and panel (B) shows thyroid hormones. Effect estimates represent the change in gestational age at birth (in days) with an interquartile range increase in hormone concentration. Red and blue estimates correspond to female and male fetuses, respectively. Squares denote effects from hormones at visit 1 , while circles denote effects from hormones at visit 3.

A

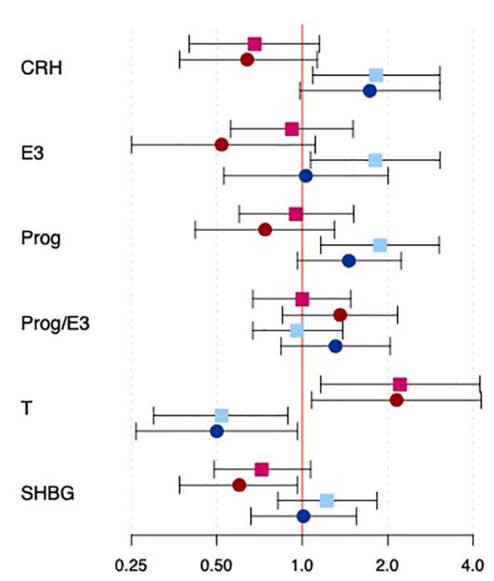

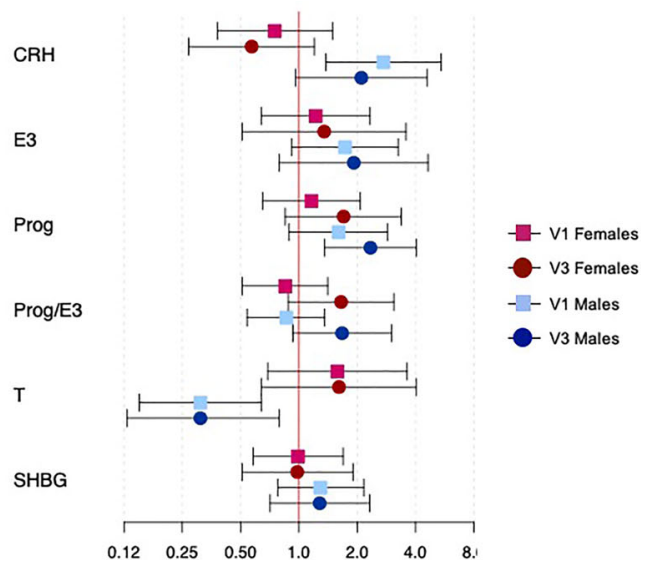

FIGURE 2 | Associations between CRH and reproductive hormone concentrations and binary outcomes of overall and spontaneous PTB, specific to fetal sexes and study visit. Panel (A) shows overall PTB and panel (B) shows spontaneous PTB. Effect estimates represent the odds of overall or spontaneous PTB with an interquartile range increase in hormone concentration. Red and blue estimates correspond to female and male fetuses, respectively. Squares denote effects from hormones at visit 1 , while circles denote effects from hormones at visit 3.

outcomes at both study visits, though only effects with hormones at 18 weeks among male fetuses were significant (overall PTB OR: 1.82, 95\% CI: 1.09, 3.05; spontaneous PTB OR: 2.73, 95\% CI: $1.38,5.43)$. Fetal sex also modified the association between estriol and overall PTB (interaction $\mathrm{p}=0.022$ ); female fetuses saw a marginal reduction in the odds of PTB with an IQR increase in estriol at 26 weeks (OR: 0.52, 95\% CI: 0.25, 1.11), while male fetuses saw an increase in odds of PTB with an IQR increase in estriol at 18 weeks (OR: 1.81, 95\% CI: 1.07, 3.06). Results for progesterone were similar; fetal sex modified only associations with overall PTB (interaction $\mathrm{p}=0.011$ ), and significant increased odds of PTB were observed for male fetuses at 18 weeks (OR: 1.88, 95\% CI: 1.16, 3.04). Though effect estimates were not significant, study visit was important for determining the associations between the ratio of progesterone to estriol and both overall and spontaneous PTB; effect estimates were positive 
and larger in magnitude at 26 weeks compared to 18 weeks among both male and female fetuses. Testosterone showed highly significant effect modification by fetal sex for both overall and spontaneous PTB (both interaction $\mathrm{p}<0.001$ ), with an IQR increase in testosterone conferring lower risk of PTB among male fetuses and higher risk among female fetuses which did not differ between study visits. Female fetuses saw 2.21 times the odds (95\% CI: 1.16, 4.23) of overall PTB with an IQR increase in testosterone at 18 weeks, while males saw 0.52 times the odds (95\% CI: 0.30, 0.89) at the same study visit. Finally, fetal sex modified the association between SHBG and overall PTB; an IQR increase in SHBG was associated with 0.60 times the odds $(95 \%$ CI: $0.37,0.96)$ of PTB only among female fetuses at 26 weeks.

Results for exploratory analyses assessing the binary outcomes of overall and spontaneous PTB and their associations with thyroid hormones are shown in Figure 3 (effect estimates, confidence intervals, and p-values for fetal sex interaction terms can be found in Supplementary Table S2). Though most associations were not significant, effect modification by fetal sex was observed for T3 on both overall and spontaneous PTB (both interaction $\mathrm{p}=0.013$ ), and the ratio of T3 to T4 on overall PTB (interaction $\mathrm{p}=0.032$ ). An IQR increase in $\mathrm{T} 3$ at both study visits resulted in reduced odds of PTB among female fetuses and increased odds of PTB among male fetuses, though only results for spontaneous PTB among male fetuses were significant (visit 1 OR: 2.01, 95\% CI: 1.10, 3.65; visit 3 OR: $2.05,95 \%$ CI: 1.10, 3.84). An IQR increase in fT4 at both study visits resulted in marginally increased odds of overall and spontaneous PTB among only male fetuses. Lastly, an IQR increase in the ratio of T3 to T4 resulted in reduced odds of PTB among female fetuses and increased odds of PTB among male fetuses, though none of these associations reached statistical significance.

\section{DISCUSSION}

In the present analysis we explored associations between hormone concentrations measured at two time points during mid-gestation and timing of delivery. Because of previous work indicating dramatic changes in some hormone concentrations through pregnancy, as well as differential susceptibility to adverse pregnancy outcomes based on fetal sex, we determined effect estimates specific to hormones measured at 18 versus 26 weeks gestation and male versus female fetal sexes. Overall, our results suggest that some hormones, particularly reproductive hormones, have different effects on timing of delivery depending on the sex of the fetus, and most associations show male fetuses being at greater risk for early delivery. We also observed that some associations differed when hormones were assessed at 18 versus 26 weeks gestation. An exploratory analysis of the binary outcomes overall and spontaneous PTB revealed similar findings with greater significance. Interestingly, results for the spontaneous subtype of PTB were less affected by study visit than results for overall $\mathrm{PTB}$, supporting separation of these outcomes into more homogenous subgroups.

\section{CRH and Reproductive Hormones}

Reduced gestational age at birth was observed among only male fetuses with increased concentrations of $\mathrm{CRH}$ at 18 weeks. While very little epidemiologic work has been done to understand the roles of CRH in adverse birth outcomes, researchers have speculated its influence on labor and delivery for decades. Detection of elevated concentrations of CRH during the second trimester in women who eventually delivered preterm compared to those who delivered after 37 weeks was first described by Campbell et al. in 1987 (34). Since then, CRH has been described as being involved in a "placental clock," with
A

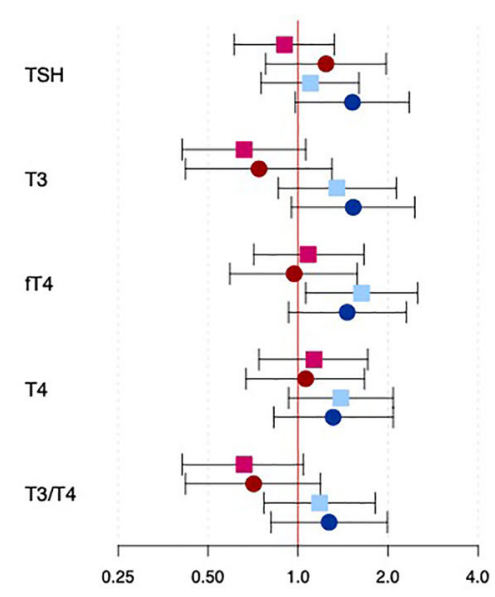

B

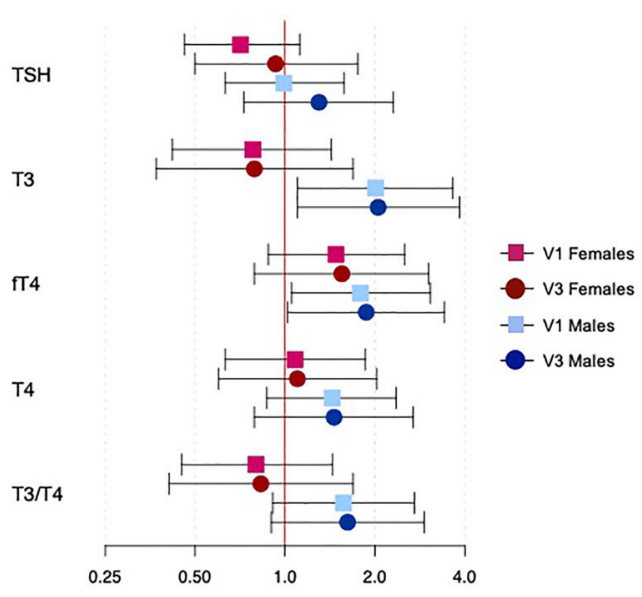

FIGURE 3 | Associations between thyroid hormone concentrations and binary outcomes of overall and spontaneous PTB, specific to fetal sexes and study visit. Panel (A) shows overall PTB and panel (B) shows spontaneous PTB. Effect estimates represent the odds of overall or spontaneous PTB with an interquartile range increase in hormone concentration. Red and blue estimates correspond to female and male fetuses, respectively. Squares denote effects from hormones at visit 1 , while circles denote effects from hormones at visit 3. 
$\mathrm{CRH}$ concentrations as early as 20 weeks possibly setting the stage for future preterm delivery (21). CRH receptors are present in the myometrium (35) and in the fetal zone of the fetal adrenal gland (36), so CRH could exert its effects on labor by interacting with these receptors.

We observed reduced gestational age, and greater odds of overall and spontaneous PTB, with increasing progesterone concentrations when fetal sex was male, but other studies demonstrating similar significant associations are lacking. One study observed progesterone concentrations measured between 28 and 32 weeks gestation to be higher among women who delivered preterm compared to full term (22). We observed higher progesterone concentrations among PTB cases when fetal sex was male, but only around 18 weeks gestation. We also observed higher progesterone concentrations around 26 weeks among women who spontaneously delivered preterm compared to women who carried to term, regardless of fetal sex.

Previous work has shown that a ratio favoring estriol in midpregnancy (23) and at delivery (37) is associated with earlier time of labor. Progesterone concentrations rise steadily during pregnancy, contributing to uterine quiescence, downregulation of prostaglandin production, and immune tolerance of the fetus $(11,12)$. At the onset of human labor, progesterone concentrations do not notably decrease; rather, the body's response to progesterone is dampened. It is not clear exactly how this occurs, but possibilities include reduction in progesterone receptor expression, changes in receptor isoforms, and local progesterone metabolism (38). As term approaches, the ratio of progesterone to estriol shifts to favor estrogens, with the functional decrease in progesterone driving initiation of labor (39). The new dominance of estrogens promotes an increase in prostaglandin and oxytocin receptors and enzymes responsible for muscle contractions, which work together to help promote labor (10). We observed a positive association between odds of PTB and estriol concentrations when fetal sex was male, but we also unexpectedly observed later gestational age at birth with higher concentrations of estriol at 26 weeks gestation when the fetus was female. In contrast with previous studies, we observed that a higher ratio of progesterone to estriol was associated with reduced gestational age at birth. Interestingly, among women who delivered preterm, a previous study observed a lower ratio of progesterone to estriol among only those without premature rupture of membranes (40), possibly implicating different endocrine pathways in the occurrence of the spontaneous subtype of PTB.

We observed significant effect modification by fetal sex on the associations between testosterone and overall and spontaneous PTB, for which the effect was positive among female fetuses and protective among male fetuses. Previous research on the effects of fetal sex on circulating maternal testosterone has been mixed. Though earlier research suggested that male fetuses conferred higher testosterone concentrations in maternal plasma during gestation $(27,41)$, more recent findings suggest that there is no significant difference (42-45). Based on more recent evidence, we believe that the fetal sex differences we have observed for testosterone are not due to elevated fetal testosterone in males impacting maternal testosterone. Nevertheless, it remains unclear what biological mechanisms may be differentially at play between fetal sexes. One group has proposed that high concentrations of testosterone may act on the endometrium to disrupt its production of secretory proteins, which may result in elevated risk for preterm delivery (8), but how this process may differ between male and female fetuses is unknown.

\section{Thyroid Hormones}

Decreased odds of PTB have been shown with increased concentrations of fT4 in the second (24) and third (46) trimesters, which contradicts our finding that fT4 was inversely associated with gestational age at birth and positively associated with odds of overall and spontaneous PTB. One prior study also found increased odds of PTB with greater T3 concentrations at 10 and 26 weeks gestation (46). Similarly, we found that T3 was associated with increased odds of spontaneous PTB when the fetus was male. Mechanisms of the association between thyroid hormones and PTB are poorly understood, but previous research has suggested that altered thyroid hormone concentrations may be involved in other disease states or exposures for which we have evidence of associations with PTB, such as oxidative stress and inflammation (47-49), or environmental exposures such as phthalates (50-52).

Thyroid hormones are critical for proper brain and skeletal development of the fetus (13). The fetus requires maternal thyroid hormones in the first half of pregnancy, during which time the fetal thyroid gland is immature and cannot produce its own hormones (14). During the second half of pregnancy, both maternal and fetal thyroid hormones are at play. Thyroid hormones cross the placenta via thyroid hormone transporters (15), and differential expression of these transporters can affect the levels of thyroid hormones available to the fetus. Thus, assessment of expression patterns of hormone transporters may be necessary to fully understand thyroid hormone associations with timing of labor.

Several previous studies have observed that male fetal sex is associated with a greater risk of delivering preterm. Proposed biological explanations for this observation include a proinflammatory environment generated by a male fetus (25) and larger size at birth for males relative to females (26). We observed significant associations with timing of labor unique to women carrying a male fetus for $\mathrm{CRH}$, estriol, progesterone, and fT4, providing further evidence that the effect of fetal sex on the timing of labor is complex, possibly involving diverse endocrine pathways.

\section{Strengths and Limitations}

The present study was subject to several limitations. We were not able to measure hCG, for which previous work has suggested a fetal sex difference is present (53). We also did not assess thyroid autoantibody status which could be clouding our thyroid hormone results due to unmeasured confounding. Some critical changes in the maternal endocrine environment occur later in gestation than we were able to measure, such as the exponential increase in $\mathrm{CRH}$ right before the onset of labor. Although the goal of this study was to determine whether mid- 
pregnancy hormone levels were indicative of increased risk of adverse pregnancy outcomes, measurements at later time points could shed additional light on the various endocrine pathways implicated in adverse birth outcomes. We observed low rates of the spontaneous subtype of PTB, which reduces the reliability of effect estimates, particularly when investigating effects specific to study visit and fetal sex. However, these lower rates may have been observed because we excluded women with preexisting conditions from our cohort to allow more precise examination of associations between hormone concentrations and birth outcomes, since preexisting conditions can influence hormone concentrations and susceptibility to adverse birth outcomes. Furthermore, excluding women with preexisting conditions may limit the generalizability of our findings.

Despite the aforementioned limitations, this study was also strong in various ways. This is one of few studies to assess a broad panel of hormone concentrations in relation to gestational age, which is advantageous because the disease state may implicate multiple endocrine pathways. This is also one offew studies to assess hormones at more than one time point during gestation to investigate different windows of susceptibility to hormonal disruption. Assessment of hormones at multiple time points is also necessary for hormones whose concentrations change dramatically through pregnancy, including $\mathrm{CRH}$, estriol, and progesterone. We are also one of few groups to assess interactions between gestational hormone concentrations and fetal sex, despite existing research suggesting differential susceptibility to early delivery between male and female fetuses. Finally, our study was strengthened by a higher sample size of mothers than was seen in most previously published cohorts, which is particularly important when studying rare outcomes such as spontaneous PTB.

In conclusion, we observed a range of associations between hormones and timing of delivery. We found differences based on the timing of hormone assessment, and many significant findings were unique to mothers carrying a male fetus. Future work will attempt to place these findings in the context of relevant environmental contaminants on the island of Puerto Rico by exploring possibilities of endocrine disruption as a mediator between chemical exposures and pregnancy outcomes. Additional studies are needed to more fully elucidate the role of altered hormone concentrations in the etiology of adverse birth outcomes.

\section{DATA AVAILABILITY STATEMENT}

The raw data supporting the conclusions of this article will be made available by the authors, without undue reservation.

\section{REFERENCES}

1. Blencowe H, Cousens S, Oestergaard MZ, Chou D, Moller A-B, Narwal R, et al. National, Regional, and Worldwide Estimates of Preterm Birth Rates in the Year 2010 With Time Trends Since 1990 for Selected Countries: A Systematic Analysis and Implications. Lancet (London England) (2012) 379:2162-72. doi: 10.1016/S0140-6736(12)60820-4

\section{ETHICS STATEMENT}

This study was approved by the research and ethics committees of the University of Michigan School of Public Health, University of Puerto Rico, Northeastern University, and participating hospitals and clinics. The patients/participants provided their written informed consent to participate in this study.

\section{AUTHOR CONTRIBUTIONS}

$\mathrm{AC}$ analyzed and interpreted data and drafted the original article. JM, JC, AA, RL-C, and CV made substantial contributions to the conception and design of the study. DW and ZR contributed to acquisition of data. BM, RL-C, and MO'n assisted with interpretation of data. All authors contributed to the article and approved the submitted version.

\section{FUNDING}

This work was supported by grant number P42ES017198 from the NIH; grant numbers R01ES031591, R01ES032203, and P30ES017885 from the NIEHS; and program grant number UH3OD023251 for the Environmental influences on Child Health Outcomes (ECHO) from the NIH, Office of the Director.

\section{ACKNOWLEDGMENTS}

We would like to extend our gratitude to all PROTECT study participants and their families. The authors also thank the nurses and research staff who participated in cohort recruitment and follow up, as well as the Federally Qualified Health Centers (FQHC) in Puerto Rico that facilitated participant recruitment, including Morovis Community Health Center, Prymed in Ciales, Camuy Health Services, Inc. and the Delta OBGyn Group in Manati, as well as the Manati Medical Center and the Metro Pavia Hospital in Arecibo.

\section{SUPPLEMENTARY MATERIAL}

The Supplementary Material for this article can be found online at: https://www.frontiersin.org/articles/10.3389/fendo.2021. 742145/full\#supplementary-material

2. Martin JA, Hamilton BE, Osterman MJKS, Driscoll AK, Drake P. Births: Final Data for 2016 National Vital Statistics Reports. Natl Vital Stat Rep (2018) 67:1-55.

3. Luyckx VA. Preterm Birth and its Impact on Renal Health. Semin Nephrol (2017) 37:311-9. doi: 10.1016/j.semnephrol.2017.05.002

4. Leung MP, Thompson B, Black J, Dai S, Alsweiler JM. The Effects of Preterm Birth on Visual Development. Clin Exp Optom (2018) 101:4-12. doi: 10.1111/ cxo. 12578 
5. Spittle AJ, Morgan C, Olsen JE, Novak I, Cheong JLY. Early Diagnosis and Treatment of Cerebral Palsy in Children With a History of Preterm Birth. Clin Perinatol (2018) 45:409-20. doi: 10.1016/j.clp.2018.05.011

6. Schubert U, Muller M, Abdul-Khaliq H, Norman M. Preterm Birth Is Associated With Altered Myocardial Function in Infancy. J Am Soc Echocardiogr (2016) 29:670-8. doi: 10.1016/j.echo.2016.03.011

7. McElrath TF, Hecht JL, Dammann O, Boggess K, Onderdonk A, Markenson G, et al. Pregnancy Disorders That Lead to Delivery Before the 28th Week of Gestation: An Epidemiologic Approach to Classification. Am J Epidemiol (2008) 168:980-9. doi: 10.1093/aje/kwn202

8. Ruge S, Sørensen S, Pedersen JF, Lange AP, Byrjalsen I, Bohn H. Secretory Endometrial Protein PP14 in Women With Early Pregnancy Bleeding. Hum Reprod (1991) 6:885-8. doi: 10.1093/oxfordjournals.humrep.a137445

9. Okon MA, Laird SM, Tuckerman EM, Li TC. Serum Androgen Levels in Women Who Have Recurrent Miscarriages and Their Correlation With Markers of Endometrial Function. Fertil Steril (1998) 69:682-90. doi: 10.1016/s0015-0282 (98)00007-7

10. Challis JRG, Matthews SG, Gibb W, Lye SJ. C DPJRG. Endocrine and Paracrine Regulation of Birth at Term and Preterm. Endocr Rev (2000) 21:514-50. doi: 10.1210/edrv.21.5.0407

11. Carlo Di Renzo G, Giardina I, Clerici G, Brillo E, Gerli S. Progesterone in Normal and Pathological Pregnancy. Horm Mol Biol Clin Investig (2016) 27:35-48. doi: 10.1515/hmbci-2016-0038

12. Byrns MC. Regulation of Progesterone Signaling During Pregnancy: Implications for the Use of Progestins for the Prevention of Preterm Birth. J Steroid Biochem Mol Biol (2014) 139:173-81. doi: 10.1016/j.jsbmb.2013.01.015

13. Yen PM. Physiological and Molecular Basis of Thyroid Hormone Action. Physiol Rev (2001) 81:1097-142. doi: 10.1152/physrev.2001.81.3.1097

14. Springer D, Jiskra J, Limanova Z, Zima T, Potlukova E. Thyroid in Pregnancy: From Physiology to Screening. Crit Rev Clin Lab Sci (2017) 54:102-16. doi: 10.1080/10408363.2016.1269309

15. Forhead AJ, Fowden AL. Thyroid Hormones in Fetal Growth and Prepartum Maturation. J Endocrinol (2014) 221:R87-R103. doi: 10.1530/JOE-14-0025

16. Mannisto T, Mendola P, Grewal J, Xie Y, Chen Z, Laughon SK. Thyroid Diseases and Adverse Pregnancy Outcomes in a Contemporary US Cohort. J Clin Endocrinol Metab (2013) 98:2725-33. doi: 10.1210/jc.2012-4233

17. Allan WC, Haddow JE, Palomaki GE, Williams JR, Mitchell ML, Hermos RJ, et al. Maternal Thyroid Deficiency and Pregnancy Complications: Implications for Population Screening. J Med Screen (2000) 7:127-30. doi: 10.1136/jms.7.3.127

18. Abalovich M, Gutierrez S, Alcaraz G, Maccallini G, Garcia A, Levalle O. Overt and Subclinical Hypothyroidism Complicating Pregnancy. Thyroid (2002) 12:63-8. doi: 10.1089/105072502753451986

19. Nazarpour S, Ramezani Tehrani F, Simbar M, Azizi F. Thyroid Dysfunction and Pregnancy Outcomes. Iran J Reprod Med (2015) 13:387-96.

20. Smith R, Nicholson RC. Corticotrophin Releasing Hormone and the Timing of Birth. Front Biosci (2007) 12:912-8. doi: 10.2741/2113

21. McLean M, Bisits A, Davies J, Woods R, Lowry P, Smith R. A Placental Clock Controlling the Length of Human Pregnancy. Nat Med (1995) 1:460-3. doi: $10.1038 / \mathrm{nm} 0595-460$

22. Feng T, Allen JC, Ng MJ, Yeo GSH, Kwek KYC, Chern BSM, et al. The Association Between Serum Progesterone Level and Preterm Delivery. Int $J$ Gynaecol Obstet (2018) 142:308-14. doi: 10.1002/ijgo.12564

23. Ruiz RJ, Saade GR, Brown CEL, Nelson-Becker C, Tan A, Bishop S, et al. The Effect of Acculturation on Progesterone/Estriol Ratios and Preterm Birth in Hispanics. Obstet Gynecol (2008) 111:309-16. doi: 10.1097/ 01.AOG.0000297896.00491.2c

24. Korevaar TIM, Chaker L, Medici M, de Rijke YB, Jaddoe VWV, Steegers EAP, et al. Maternal Total T4 During the First Half of Pregnancy: Physiologic Aspects and the Risk of Adverse Outcomes in Comparison With Free T4. Clin Endocrinol (Oxf) (2016) 85:757-63. doi: 10.1111/cen.13106

25. Challis J, Newnham J, Petraglia F, Yeganegi M, Bocking A. Fetal Sex and Preterm Birth. Placenta (2013) 34:95-9. doi: 10.1016/j.placenta.2012.11.007

26. McGregor JA, Leff M, Orleans M, Baron A. Fetal Gender Differences in Preterm Birth: Findings in a North American Cohort. Am J Perinatol (1992) 9:43-8. doi: 10.1055/s-2007-994668

27. Meulenberg PM, Hofman JA. Maternal Testosterone and Fetal Sex. J Steroid Biochem Mol Biol (1991) 39:51-4. doi: 10.1016/0960-0760(91)90012-t
28. Bremme K, Eneroth P. Fetal Sex Dependent Hormone Levels in Early Pregnant Women With Elevated Maternal Serum Alpha-Fetoprotein. Int $J$ Gynaecol Obstet Off Organ Int Fed Gynaecol Obstet (1983) 21:451-7. doi: 10.1016/0020-7292(83)90034-6

29. Takagi S, Yoshida T, Tsubata K, Ozaki H, Fujii TK, Nomura Y, et al. Sex Differences in Fetal Gonadotropins and Androgens. J Steroid Biochem (1977) 8:609-20. doi: 10.1016/0022-4731(77)90270-9

30. Meeker JD, Cantonwine DE, Rivera-González LO, Ferguson KK, Mukherjee B, Calafat AM, et al. Distribution, Variability, and Predictors of Urinary Concentrations of Phenols and Parabens Among Pregnant Women in Puerto Rico. Environ Sci Technol (2013) 47:3439-47. doi: 10.1021/es400510g

31. Dietrich JW, Landgrafe G, Fotiadou EH. TSH and Thyrotropic Agonists: Key Actors in Thyroid Homeostasis. J Thyroid Res (2012) 2012:351864. doi: $10.1155 / 2012 / 351864$

32. Romero R, Scoccia B, Mazor M, Wu YK, Benveniste R. Evidence for a Local Change in the Progesterone/ Estrogen Ratio in Human Parturition at Term. Am J Obstet Gynecol (1988) 159:657-60. doi: 10.1016/S0002-9378(88)80029-2

33. Pettker C, Goldberg J, El-Sayed Y, Copel J. Committee Opinion No 700: Methods for Estimating the Due Date. Obstet Gynecol (2017) 129:e150-4. doi: 10.1097/AOG.0000000000002046

34. Campbell EA, Linton EA, Wolfe CD, Scraggs PR, Jones MT, Lowry PJ. Plasma Corticotropin-Releasing Hormone Concentrations During Pregnancy and Parturition. JClin Endocrinol Metab (1987) 64:1054-9. doi: 10.1210/jcem-64-5-1054

35. Grammatopoulos DK. The Role of CRH Receptors and Their Agonists in Myometrial Contractility and Quiescence During Pregnancy and Labour. Front Biosci (2007) 12:561-71. doi: 10.2741/2082

36. Smith R, Mesiano S, Chan EC, Brown S, Jaffe RB. Corticotropin-Releasing Hormone Directly and Preferentially Stimulates Dehydroepiandrosterone Sulfate Secretion by Human Fetal Adrenal Cortical Cells. J Clin Endocrinol Metab (1998) 83:2916-20. doi: 10.1210/jcem.83.8.5020

37. Moran D, Mcgarrigle HH, Lachelin GC. Lack of Normal Increase in Saliva Estriol / Progesterone Ratio in Women With Labor Induced at 42 Weeks ' Gestation. Am J Obstet Gynecol (1992) 167(6):1563-4. doi: 10.1016/0002-9378(92)91740-2

38. Mesiano S, Welsh TN. Steroid Hormone Control of Myometrial Contractility and Parturition. Semin Cell Dev Biol (2007) 18:321-31. doi: 10.1016/ j.semcdb.2007.05.003

39. Snegovskikh V, Park JS. Endocrinology of Parturition. Endocrinol Metab Clin North Am (2006) 35:173-91. doi: 10.1016/j.ecl.2005.09.012

40. Darne J, Mcgarrigle H, Lachelin G. Increased Saliva Oestriol to Progesterone Ratio Before Idiopathic Preterm Delivery: A Possible Predictor for Preterm Labour? Br Med J (1987) 294:270-2. doi: 10.1136/bmj.294.6567.270

41. Klinga K, Bek E, Runnebaum B. Maternal Peripheral Testosterone Levels During the First Half of Pregnancy. Am J Obstet Gynecol (1978) 131:60-2. doi: 10.1016/0002-9378(78)90474-x

42. Voegtline KM, Costigan KA, Kivlighan KT, Henderson JL, DiPietro JA. SexSpecific Associations of Maternal Prenatal Testosterone Levels With Birth Weight and Weight Gain in Infancy. J Dev Orig Health Dis (2013) 4:280-4. doi: $10.1017 /$ S2040174413000135

43. Toriola AT, Vääräsmäki M, Lehtinen M, Zeleniuch-Jacquotte A, Lundin E, Rodgers KG, et al. Determinants of Maternal Sex Steroids During the First Half of Pregnancy. Obstet Gynecol (2011) 118:1029-36. doi: 10.1097/AOG.0b013e3182342b7f

44. Cohen-Bendahan CCC, van Goozen SHM, Buitelaar JK, Cohen-Kettenis PT. Maternal Serum Steroid Levels are Unrelated to Fetal Sex: A Study in Twin Pregnancies. Twin Res Hum Genet Off J Int Soc Twin Stud (2005) 8:173-7. doi: $10.1375 / 1832427053738764$

45. Vlková B, Vávrová S, Szemes T, Minárik G, Turna J, Celec P. Testosterone and Estradiol in Maternal Plasma and Their Relation to Fetal Sex. Prenat Diagn (2010) 30:806-7. doi: 10.1002/pd.2535

46. Johns LE, Ferguson KK, McElrath TF, Mukherjee B, Seely EW, Meeker JD. Longitudinal Profiles of Thyroid Hormone Parameters in Pregnancy and Associations With Preterm Birth. PloS One (2017) 12:1-15. doi: 10.1371/ journal.pone. 0169542

47. Mancini A, Di Segni C, Raimondo S, Olivieri G, Silvestrini A, Meucci E, et al. Thyroid Hormones, Oxidative Stress, and Inflammation. Mediators Inflammation (2016) 2016:6757154. doi: 10.1155/2016/6757154

48. Moore TA, Ahmad IM, Zimmerman MC. Oxidative Stress and Preterm Birth: An Integrative Review. Biol Res Nurs (2018) 20:497-512. doi: 10.1177/ 1099800418791028 
49. Cappelletti M, Della Bella S, Ferrazzi E, Mavilio D, Divanovic S. Inflammation and Preterm Birth. J Leukoc Biol (2016) 99:67-78. doi: 10.1189/jlb.3MR0615-272RR

50. Cathey AL, Watkins D, Rosario ZY, Velez C, Alshawabkeh AN, Cordero JF, et al. Associations of Phthalates and Phthalate Replacements With $\mathrm{CRH}$ and Other Hormones Among Pregnant Women in Puerto Rico. J Endocr Soc (2019) 3:1127-49. doi: 10.1210/js.2019-00010

51. Johns LE, Ferguson KK, McElrath TF, Mukherjee B, Meeker JD. Associations Between Repeated Measures of Maternal Urinary Phthalate Metabolites and Thyroid Hormone Parameters During Pregnancy. Environ Health Perspect (2016) 124:1808-15. doi: 10.1289/EHP170

52. Ferguson KK, Rosen EM, Rosario Z, Feric Z, Calafat AM, McElrath TF, et al. Environmental Phthalate Exposure and Preterm Birth in the PROTECT Birth Cohort. Environ Int (2019) 132:105099. doi: 10.1016/j.envint.2019.105099

53. Adibi JJ, Lee MK, Saha S, Boscardin WJ, Apfel A, Currier RJ. Fetal Sex Differences in Human Chorionic Gonadotropin Fluctuate by Maternal Race, Age, Weight and by Gestational Age. J Dev Orig Health Dis (2015) 6:493-500. doi: $10.1017 /$ S2040174415001336
Conflict of Interest: The authors declare that the research was conducted in the absence of any commercial or financial relationships that could be construed as a potential conflict of interest.

Publisher's Note: All claims expressed in this article are solely those of the authors and do not necessarily represent those of their affiliated organizations, or those of the publisher, the editors and the reviewers. Any product that may be evaluated in this article, or claim that may be made by its manufacturer, is not guaranteed or endorsed by the publisher.

Copyright ( 2021 Cathey, Watkins, Rosario, Vega, Mukherjee, O’Neill, Loch-Caruso, Alshawabkeh, Cordero and Meeker. This is an open-access article distributed under the terms of the Creative Commons Attribution License (CC BY). The use, distribution or reproduction in other forums is permitted, provided the original author $(s)$ and the copyright owner(s) are credited and that the original publication in this journal is cited, in accordance with accepted academic practice. No use, distribution or reproduction is permitted which does not comply with these terms. 